\title{
The lasting effects of childhood trauma
}

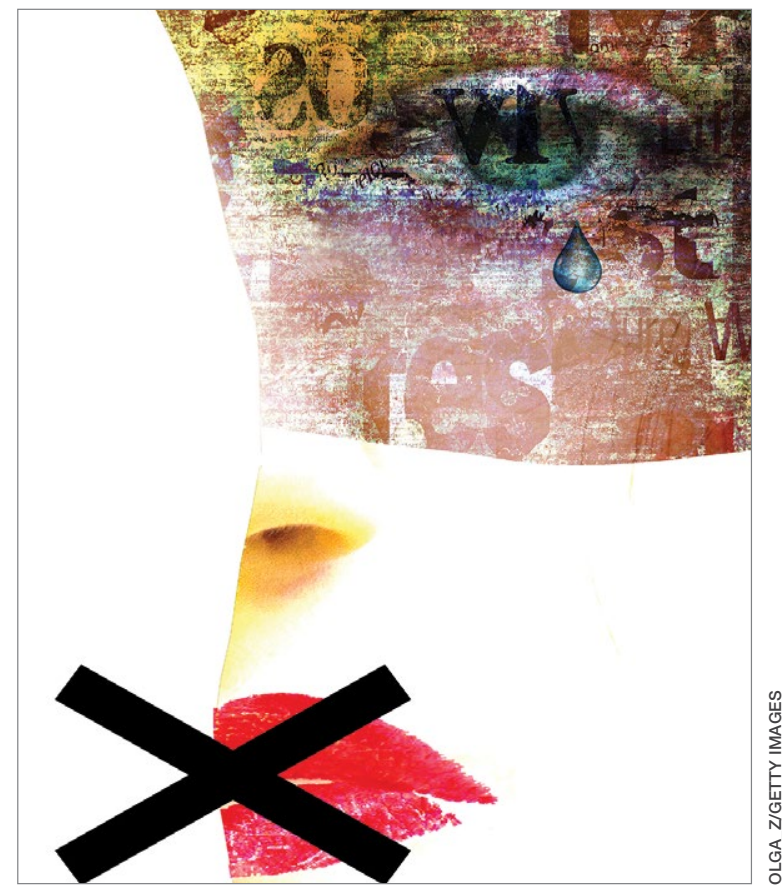

Shikha Verma, MD, FAPA

Medical Director, Northern California

Evolve Treatment Centers

Danville, California

Assistant Professor

Department of Psychiatry and Behavioral Health

Rosalind Franklin University of Medicine and Science

North Chicago, Illinois

\section{Ruchita Agrawal, MD, FAPA}

Associate Chief Medical Officer, Adult Services

Seven Counties Services

Louisville, Kentucky

\section{Evidence-based interventions can help address PTSD, other symptoms in adulthood}

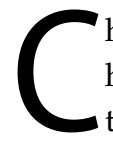

hildhood trauma, which is also called adverse childhood experiences (ACEs), can have lasting detrimental effects on individuals as they grow and mature into adulthood. ACEs may occur in children age $\leq 18$ years if they experience abuse or neglect, violence, or other traumatic losses. More than $60 \%$ of people experience at least $1 \mathrm{ACE}$, and 1 in 6 individuals reported that they had experienced $\geq 4$ ACEs. ${ }^{1}$ Subsequent additional ACEs have a cumulative deteriorating impact on the brain. This predisposes individuals to mental health disorders, substance use disorders, and other psychosocial problems. The efficacy of current therapeutic approaches provides only partial symptom resolution. For such individuals, the illness load and health care costs typically remain high across the lifespan. ${ }^{1,2}$

In this article, we discuss types of ACEs, protective factors and risk factors that influence the development of posttraumatic stress disorder (PTSD) in individuals who experience ACEs, how ACEs can negatively impact mental health in adulthood, and approaches to prevent or treat PTSD and other symptoms.

\section{Types of trauma and correlation with PTSD}

ACEs can be indexed as neglect or emotional, physical, or sexual abuse. Physical and sexual abuse strongly correlate with an increased risk of PTSD. ${ }^{3}$ Although neglect and emotional abuse do not directly predict the development of PTSD, these

The authors report no financial relationships with any companies whose products are mentioned in this article, or with manufacturers of competing products.

doi: 10.12788/cp.0101 
experiences foretell high rates of lifelong trauma exposure and are indirectly related to late PTSD symptoms. ${ }^{4,5}$ ACEs can impede an individual's cognitive, social, and emotional development, diminish quality of life, and lead to an early death. ${ }^{6}$ The lifetime prevalence of PTSD is $6.1 \%$ to $9.2 \% .^{7}$ Compared with men, women are 4 times more likely to develop PTSD following a traumatic event. ${ }^{7}$

The development of PTSD is influenced by the nature, duration, and degree of trauma, and age at the time of exposure to trauma. Children who survive complex trauma ( $\geq 2$ types of trauma) have a higher likelihood of developing PTSD. ${ }^{8}$ Prolonged trauma exposure has a more substantial negative impact than a one-time occurrence. However, it is an erroneous oversimplification to assume that each type of ACE has an equally traumatic effect. ${ }^{6}$

\section{Factors that protect against PTSD}

Factors that can protect against developing PTSD are listed in Table 1. ${ }^{7}$ Two of these are resilience and hope.

Resilience is defined as an individual's strength to cope with difficulties in life. ${ }^{9}$ Resilience has internal psychological characteristics and external factors that aid in protecting against childhood adversities. ${ }^{10,11}$ The Brief Resilience Scale is a self-assessment that measures innate abilities to cope, including optimism, self-efficacy, patience, faith, and humor. ${ }^{12,13}$ External factors associated with resilience are family, friends, and community support. ${ }^{11,13}$

Hope can help in surmounting ACEs. The Adult Hope Scale has been used in many studies to assess this construct in individuals who have survived trauma. ${ }^{13}$ Some studies have found decreased hope in individuals who sustained early trauma and were diagnosed with PTSD in adulthood. ${ }^{14}$ A study examining children exposed to domestic violence found that children who showed high hope, endurance, and curiosity were better able to cope with adversities. ${ }^{15}$

\section{PTSD risk factors}

Many individual and societal risk factors can influence the likelihood of developing

\section{Table 1}

\section{PTSD: Protective factors and risk factors}

Protective factors

Being male (more men than women are exposed to trauma, but PTSD is twice as common in women)

A strong relationship with family and peers

Resilience and hope

Limited genetic predisposition to psychiatric illness

Risk factors

Severity and duration of trauma

Age at trauma

Type and number of traumatic experiences

Severity of initial response to trauma

General childhood adversity

Reported childhood abuse

Gender

Low socioeconomic status

Low education

First-degree relative with a history of depression, other psychiatric illness, or substance use

Single, divorced, widowed, or socially withdrawn

Inadequate family and peer support

PTSD: posttraumatic stress disorder

Source: Reference 7

PTSD. Some of these factors are outlined in Table 1. ${ }^{7}$

\section{Pathophysiology of PTSD}

Multiple brain regions, pathways, and neurotransmitters are involved in the development of PTSD. Neuroimaging has identified volume and activity changes of the hippocampus, prefrontal cortex, and amygdala in patients with early trauma and PTSD. Some researchers have suggested a gross reduction in locus coeruleus neuronal volume in war veterans with a likely diagnosis of PTSD compared with controls. ${ }^{16,17}$ In other studies, chronic stress exposure has been found to cause neuronal cell death and affect neuronal plasticity in the limbic area of the brain. ${ }^{18}$

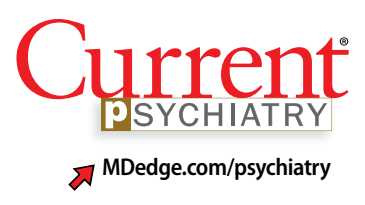

Clinical Point

ACEs can impede cognitive, social, and emotional development, diminish quality of life, and lead to an early death

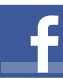

Discuss this article at www.facebook.com/ MDedgePsychiatry 


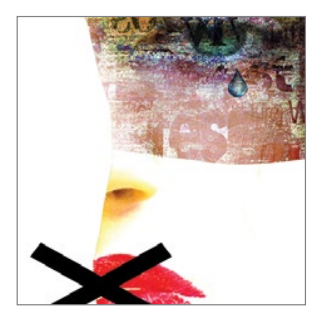

Lasting effects of childhood trauma

Clinical Point

More than $30 \%$

of individuals

who experience

adverse childhood

experiences

develop PTSD

\section{DSM-5 criteria for posttraumatic stress disorder}

Trauma exposure

\begin{tabular}{l|l} 
Trauma & Actual or threatened violent death, serious injury or accident, or sexual violence \\
A. Exposure & $\begin{array}{l}\text { Via any of the following: } \\
\text { 1. Directly exposed to trauma }\end{array}$ \\
$\begin{array}{l}\text { 2. Eyewitness (in person) to others directly exposed to trauma } \\
\text { 3. Learning of direct exposure to trauma of a close family member or close friend } \\
\text { 4. Repeated or extreme exposure to aversive details of traumatic event (eg, trauma } \\
\text { workers viewing human remains or repeatedly exposed to details of child abuse), } \\
\text { in person or via work-related electronic media }\end{array}$
\end{tabular}

Symptom groups $B$ to $E$ (symptoms beginning or worsening after the traumatic event)

B. Intrusion $\geq 1$ intrusion symptoms:

1. Recurrent, involuntary, distressing trauma memories

2. Recurrent, distressing trauma-related dreams

3. Dissociative reactions/flashbacks related to trauma

4. Intense or prolonged psychological distress to trauma reminders

5. Marked physiological reactions to trauma reminders

C. Avoidance $\geq 1$ avoidance symptoms:

1. Avoidance of or efforts to avoid distressing internal trauma reminders (memories, thoughts, feelings)

2. Avoidance of or efforts to avoid distressing external trauma reminders (people, places, activities)

\section{Negative $\geq 2$ negative cognition/mood symptoms:}

cognition $\quad 1$ Amnesia for important parts of trauma exposure

and mood 2 . Persistent, exaggerated negative beliefs about self, others, or the world

3. Persistent, distorted trauma-related cognitions leading to inappropriate blame of self/others

4. Persistent negative emotional state (eg, fear, horror, anger, guilt, shame)

5. Loss of interest or participation in significant activities

6. Feelings of detachment or estrangement from others

7. Persistent loss of positive emotions (eg, happiness, satisfaction, love)

E. Hyperarousal $\geq 2$ marked alterations in trauma-related arousal and reactivity:

1. Irritability and angry outbursts with little/no provocation (eg, verbal/physical aggression toward people/objects)

2. Reckless or self-destructive behavior

3. Hypervigilance

4. Exaggerated startle

5. Concentration problems

6. Sleep disturbance (eg, difficulty falling or staying asleep, restless sleep)

Additional criteria

\begin{tabular}{l:l} 
F. Duration & $>1$ month \\
\hline $\begin{array}{l}\text { G. Distress/ } \\
\text { impairment }\end{array}$ & $\begin{array}{l}\text { Clinically significant distress; social/occupational/other important functioning } \\
\text { impairment }\end{array}$ \\
\hline $\begin{array}{l}\text { H. Not } \\
\text { attributable } \\
\text { to another } \\
\text { disorder }\end{array}$ & $\begin{array}{l}\text { andependent of physiological effects of a substance (eg, medical condition, alcohol) or } \\
\text { PTSD: posttraumatic stress disorder }\end{array}$ \\
$\begin{array}{l}\text { Source: Reference } 20 \\
\end{array}$
\end{tabular}

\section{Diagnosing PTSD}

More than $30 \%$ of individuals who experience ACEs develop PTSD. ${ }^{19}$ The DSM-5 diagnostic criteria for PTSD are outlined in Table $2 .^{20}$ Several instruments are used to determine the diagnosis and assess the severity of PTSD. These include the
Clinician-Administered PTSD Scale for DSM- $5,{ }^{21}$ which is a 30 -item structured interview that can be administered in 45 to 60 minutes; the PTSD Symptom Scale Self-Report Version, which is a 17-item, Likert scale, self-report questionnaire; and the Structured Clinical Interview: 
PTSD Module, which is a semi-structured interview that can take up to several hours to administer. ${ }^{21}$

Other disorders. In addition to PTSD, individuals with ACEs are at high risk for other mental health issues throughout their lifetime. Individuals with ACE often experience depressive symptoms (approximately $40 \%$ ); anxiety (approximately $30 \%$ ); anger; guilt or shame; negative self-cognition; interpersonal difficulties; rumination; and thoughts of self-harm and suicide. ${ }^{22}$ Epidemiological studies suggest that patients who experience childhood sexual abuse are more likely to develop mood, anxiety, and substance use disorders in adulthood. ${ }^{23,24}$

\section{Psychotherapeutic treatments for PTSD}

Cognitive-behavioral therapy (CBT) addresses the relationship between an individual's thoughts, emotions, and behaviors. CBT can be used to treat adults and children with PTSD. Before starting CBT, assess the patient's current safety to ensure that they have the coping skills to manage distress related to their ACEs, and address any coexisting substance use. ${ }^{25}$

According to the American Psychological Association, several CBT-based psychotherapies are recommended for treating PTSD ${ }^{26}$ :

Trauma-focused-CBT includes psychoeducation, trauma narrative, processing, exposure, and relaxation skills training. It consists of approximately 12 to 16 sessions and incorporates elements of family therapy.

Cognitive processing therapy (CPT) focuses on helping patients develop adaptive cognitive domains about the self, the people around them, and the world. CPT therapists assist in information processing by accessing the traumatic memory and trying to eliminate emotions tied to it. ${ }^{25,27}$ CPT consists of 12 to 16 structured individual, group, or combined sessions.

Prolonged exposure (PE) targets fear-related emotions and works on the principles of habituation to extinguish trauma and fear response to the trigger. This increases self-reliance and competence and decreases the generalization of anxiety to innocuous triggers. PE typically consists of 9 to 12 sessions. PE alone or in combination with cognitive restructuring is successful in treating patients with PTSD, but cognitive restructuring has limited utility in young children..$^{25,27}$

Cognitive exposure can be individual or group therapy delivered over 3 months, where negative self-evaluation and traumatic memories are challenged with the goal of interrupting maladaptive behaviors and thoughts. ${ }^{27}$

Stress inoculation training (SIT) provides psychoeducation, skills training, role-playing, deep muscle relaxation, paced breathing, and thought stopping. Emphasis is on coaching skills to alleviate anxiety, fear, and symptoms of depression associated with trauma. In SIT, exposures to traumatic memories are indirect (eg, role play), compared with $\mathrm{PE}$, where the exposures are direct. ${ }^{25}$

The American Psychological Association conditionally recommended several other forms for psychotherapy for treating patients with PTSD ${ }^{26}$ :

Brief eclectic psychotherapy uses CBT and psychodynamic approaches to target feelings of guilt and shame in 16 sessions. ${ }^{27}$

Narrative exposure therapy consists of 4 to 10 group sessions in which individuals provide detailed narration of the events; the focus is on self-respect and personal rights. ${ }^{27}$

Eye movement desensitization and reprocessing (EMDR) is a 6- to 12-session, 8-phase treatment that uses principles of accelerated information processing to target nonverbal expression of trauma and dissociative experiences. Patients with PTSD are suggested to have disrupted rapid eye movements. In EMDR, patients follow rhythmic movements of the therapist's hands or flashed light. This is designed to decrease stress associated with accessing trauma memories, the emotional/physiologic response from the memories, and negative cognitive distortions about self, and to replace negative cognition distortions with positive thoughts about self. 25,27

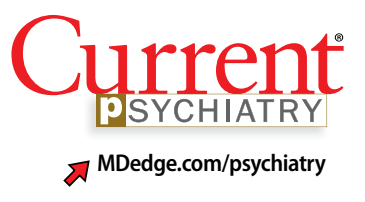

Clinical Point

Before starting CBT, ensure that your patient has the coping skills to manage distress related to their ACEs 


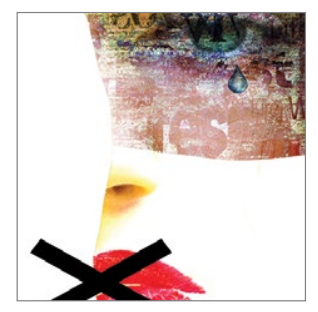

Lasting effects of childhood trauma

\section{Clinical Point}

\section{Multiple studies have found that} paroxetine, sertraline, and fluoxetine can decrease PTSD symptoms

\section{Related Resources}

- Smith P, Dalglesih T, Meiser-Stedman R. Practitioner review: posttraumatic stress disorder and its treatment in children and adolescents. J Child Psychol Psychiatry. 2019;60(5):500-515.

- North CS, Hong BA, Downs DL. PTSD: a systematic approach to diagnosis and treatment. Current Psychiatry 2018;17(4):35-43.

Drug Brand Names

Duloxetine - Cymbalta

Fluoxetine - Prozac

Mirtazapine $\cdot$ Remeron

Prazosin - Minipress

Propranolol $\cdot$ Inderal, Pronol

Sertraline • Zoloft

Paroxetine - Paxil

Venlafaxine - Effexor

Accelerated resolution therapy is a derivative of EMDR. It helps to reconsolidate the emotional and physical experiences associated with distressing memories by replacing them with positive ones or decreasing physiological arousal and anxiety related to the recall of traumatic memories. ${ }^{28}$

\section{Pharmacologic treatments Antidepressants}

Selective serotonin reuptake inhibitors (SSRIs). Multiple studies using different scales have found that paroxetine, sertraline, and fluoxetine can decrease PTSD symptoms. Approximately $60 \%$ of patients treated with SSRIs experience partial remission of symptoms, and $20 \%$ to $30 \%$ experience complete symptom resolution. ${ }^{29}$ Davidson et $\mathrm{al}^{30}$ found that $22 \%$ of patients with PTSD who received fluoxetine had a relapse of symptoms, compared with $50 \%$ of patients who received placebo.

Serotonin-norepinephrine reuptake inhibitors (SNRIs) and other antidepressants. The SNRIs venlafaxine and duloxetine can help reduce hyperarousal symptoms and improve mood, anxiety, and sleep. ${ }^{26}$ Mirtazapine, an alpha 2A/2C adrenoceptor
antagonist/5-HT 2A/2C/3 antagonist, can address PTSD symptoms from both serotonergic pathways and increase norepinephrine release by blocking autoreceptors and enhancing alpha-1 receptor activity. This alleviates hyperarousal symptoms and promotes sleep. ${ }^{29}$ In addition to having monoaminergic effects, antidepressant medications also regulate the hypothalamicpituitary-adrenal (HPA) axis response to stress and promote neurogenesis in the hippocampal region. ${ }^{29}$

\section{Adrenergic agents}

Adrenergic receptor antagonists. Prazosin, an alpha-1 adrenoceptor antagonist, decreases hyperarousal symptoms, improves sleep, and decreases nightmares related to PTSD by decreasing noradrenergic hyperactivity. ${ }^{29}$

Beta-blockers such as propranolol can decrease physiological response to trauma but have mixed results in the prevention or improvement of PTSD symptoms..$^{29,31}$

Glucocorticoid receptor agonists. In a very small study, low-dose cortisol decreased the severity of traumatic memory (consolidation phase). ${ }^{32}$ Glucocorticoid receptor agonists can also diminish memory retrieval (reconsolidation phase) through intrusive thoughts and flashbacks. ${ }^{29}$

\section{Anticonvulsants, benzodiazepines, and antipsychotics}

These medications have had a limited role in the treatment of PTSD. ${ }^{26,29}$

\section{Future directions: Preventive treatments}

Because PTSD has a profound impact on an individual's quality of life and the development of other illnesses, there is strong interest in finding treatments that can prevent

\section{Bottom Line}

Adverse childhood experiences (ACEs) are strong predictors for the development of posttraumatic stress disorder (PTSD) and other mental health or medical issues in late adolescence and adulthood. Experiencing a higher number of ACEs increases the risk of developing PTSD as an adult. Timely psychotherapeutic and pharmacologic interventions can help limit symptoms and reduce the severity of PTSD. 
PTSD. Based on limited evidence primarily from animal studies, some researchers have suggested that certain agents may someday be helpful for PTSD prevention ${ }^{29}$ :

Glucocorticoid antagonists such as corticotropin-releasing factor 1 (CRF1) antagonists or cholecystokinin 2 (CCK2) receptor antagonists might promote resilience to stress by inhibiting the HPA axis and influencing the amygdala by decreasing fear conditioning, as observed in animal models. Similarly, in animal models, CRF1 and CCK2 are predicted to decrease memory consolidation in response to exposure to stress.

Adrenoceptor antagonists and agonists also might have a role in preventive treatment, but the evidence is scarce. Prazosin, an alpha-1 adrenoceptor antagonist, was ineffective in animal models. ${ }^{29,31}$ Propranolol, a beta-adrenoceptor blocker, has had mixed results but can decrease trauma-induced physiological arousal when administered soon after exposure. ${ }^{29}$

$\mathrm{N}$-methyl-D-aspartate (NMDA) receptor antagonists. NMDA receptor function decline has also been hypothesized to decrease the reconsolidation symptoms of PTSD. ${ }^{29}$ One study examined the prevalence of PTSD in service members who were treated for burns in a military treatment center. ${ }^{33}$ The use of the NMDA receptor antagonist ketamine lowered the prevalence of PTSD among service members who were treated for burns. The suggested mechanism is preventing memory consolidation after trauma exposure. ${ }^{33}$

\section{References}

1. Centers for Disease Control and Prevention. Preventing adverse childhood experiences. Published April 3, 2020. Accessed January 26, 2021. https://www.cdc. gov/violenceprevention/childabuseandneglect/aces/ fastfact.html

2. Kessler RC, McLaughlin KA, Green JG, et al. Childhood adversities and adult psychopathology in the WHO world mental health surveys. Br J Psychiatry. 2010; 197:378-385.

3. Norman RE, Byambaa M, De R, et al. The long-term health consequences of child physical abuse, emotional abuse, and neglect: a systematic review and meta-analysis. PLoS Medicine. 2012;9(11):e1001349. doi: 10.1371/journal. pmed.1001349

4. Spertus IL, Yehuda R, Wong CM, et al. Childhood emotional abuse and neglect as predictors of psychological and physical symptoms in women presenting to a primary care practice. Child Abuse Negl. 2003;27(11):1247-1258.

5. Glück TM, Knefel M, Lueger-Schuster B. A network analysis of anger, shame, proposed ICD-11 post-traumatic stress disorder, and different types of childhood trauma in foster care settings in a sample of adult survivors.
Eur J Psychotraumatol. 2017;8(suppl 3):1372543. doi: 10.1080/20008198.2017.1372543

6. Edwards VJ, Holden GW, Felitti VJ, et al. Relationship between multiple forms of childhood maltreatment and adult mental health in community respondents: results from the adverse childhood experiences study. Am J Psychiatry. 2003;160:1453-1460.

7. Sareen J. Posttraumatic stress disorder in adults: epidemiology, pathophysiology, clinical manifestations, course, assessment, and diagnosis. UpToDate. Updated December 3, 2020. Accessed January 26, 2021. https:// www.uptodate.com/contents/posttraumatic-stressdisorder-in-adults-epidemiology-pathophysiology-clinicalmanifestations-course-assessment-and-diagnosis

8. Widom CS. Posttraumatic stress disorder in abused and neglected children grown up. Am J Psychiatry. 1999: 156;1223-1229.

9. Rutter M. Psychosocial resilience and protective mechanisms. Am J Orthopsychiatry. 1987;57(3): 316-331.

10. Ahern NR, Kiehl EM, Sole ML, et al. A review of instruments measuring resilience. Issues Compr Pediatr Nurs. 2006; 29(2):103-125.

11. Zimmerman MA. Resiliency theory: a strengths-based approach to research and practice for adolescent health. Health Educ Behav. 2013;40(4):381-383.

12. Connor KM, Davidson JR. Development of a new resilience scale: the Connor-Davidson Resilience Scale (CD-RISC). Depress Anxiety. 2003;18(2):76-82.

13. Munoz RT, Hanks H, Hellman CM. Hope and resilience as distinct contributors to psychological flourishing among childhood trauma survivors. Traumatology. 2020;26(2): 177-184.

14. Baxter MA, Hemming EJ, McIntosh HC, et al. Exploring the relationship between adverse childhood experiences and hope. J Child Sex Abus. 2017;26(8):948-956.

15. Hellman CM, Gwinn C. Camp HOPE as an intervention for children exposed to domestic violence: a program evaluation of hope, and strength of character. Child Adolesc Soc Work J. 2017;34:269-276.

16. Bracha HS, Garcia-Rill E, Mrak RE, et al. Postmortem locus coeruleus neuron count in three American veterans with probable or possible war-related PTSD. J Neuropsychiatry Clin Neurosci. 2005;17(4):503-9.

17. de Lange GM. Understanding the cellular and molecular alterations in PTSD brains: the necessity of post-mortem brain tissue. Eur J Psychotraumatol. 2017;8(1):1341824. doi: 10.1080/20008198.2017.1341824

18. Zunszain PA, Anacker C, Cattaneo A, et al. Glucocorticoids, cytokines and brain abnormalities in depression. Prog Neuropsychopharmacol Biol Psychiatry. 2011;35(3): 722-729.

19. Greeson JKP, Briggs EC, Kisiel CL, et al. Complex trauma and mental health in children and adolescents placed in foster care: findings from the national child traumatic stress network. Child Welfare. 2011;90(6):91-108.

20. Diagnostic and statistical manual of mental disorders, 5 th ed. American Psychiatric Association; 2013.

21. American Psychological Association. PTSD assessment instruments. Updated September 26, 2018. Accessed January 27, 2021. https://www.apa.org/ptsd-guideline/ assessment/

22. Bellis MA, Hughes K, Ford K, et al. Life course health consequences and associated annual costs of adverse childhood experiences across Europe and North America: a systematic review and meta-analysis. Lancet Public Health. 2019;4(10):e517-e528. doi: 10.1016/S24682667(19)30145-8

23. Mullen PE, Martin JL, Anderson JC, et al. Childhood sexual abuse and mental health in adult life. Br J Psychiatry. 1993;163:721-732.

24. Kendler KS, Bulik CM, Silberg J, et al. Childhood sexual abuse and adult psychiatric and substance use disorders in women. An epidemiological and cotwin control analysis. Arch Gen Psychiatry. 2000;57(10):953-959.

25. Chard KM, Gilman R. Counseling trauma victims: 4 brief therapies meet the test. Current Psychiatry. 2005;4(8): 50,55-58,61-62.

26. Guideline Development Panel for the Treatment of PTSD in Adults, American Psychological Association. Summary

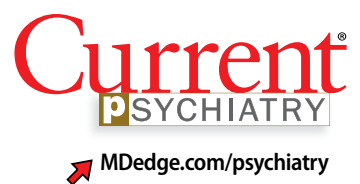

Clinical Point

Antidepressants regulate the HPA axis response to stress and promote neurogenesis in the hippocampal region 


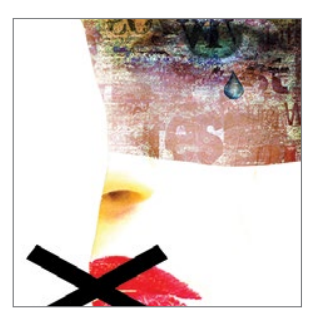

Lasting effects of childhood trauma

\section{Clinical Point}

\section{Glucocorticoid}

receptor agonists

can diminish memory retrieval of the clinical practice guideline for the treatment of posttraumatic stress disorder (PTSD) in adults. American Psychol. 2019;74(5):596-607.

27. American Psychological Association. Clinical practice guideline for the treatment of posttraumatic stress disorder. PTSD treatments. Updated June 2020. Accessed January 27, 2021. https://www.apa.org/ptsd-guideline/ treatments/

28. Kip KE, Elk CA, Sullivan KL, et al. Brief treatment of symptoms of post-traumatic stress disorder (PTSD) by use of accelerated resolution therapy (ART(®)). Behav Sci (Basel). 2012;2(2):115-134.

29. Steckler T, Risbrough V. Pharmacological treatment of PTSD - established and new approaches. Neuropharmacology. 2012;62(2):617-627.
30. Davidson JR, Connor KM, Hertzberg MA, et al. Maintenance therapy with fluoxetine in posttraumatic stress disorder: a placebo-controlled discontinuation study. J Clin Psychopharmacol. 2005;25(2):166-169.

31. Benedek DM, Friedman MJ, Zatzick D, et al. Guideline watch (March 2009): Practice guideline for the treatment of patients with acute stress disorder and posttraumatic stress disorder. Focus. 2009;7(2):204-213.

32. Aerni A, Traber R, Hock C, et al. Low-dose cortisol for symptoms of posttraumatic stress disorder. Am J Psychiat. 2004;161(8):1488-1490.

33. McGhee LL, Maani CV, Garza TH, et al. The correlation between ketamine and posttraumatic stress disorder in burned service members. J Trauma. 2008;64(2 suppl): S195-S198. doi: 10.1097/TA.0b013e318160ba1d 